\title{
Gastrointestinal Manifestation of Severe SARS-CoV-2 Infection in an Immunocompromised Dog
}

Lennon EM, ${ }^{1 *}$ Oceguera A, ${ }^{2}$ Braun E, ${ }^{3}$ Cole $\mathrm{S},{ }^{3}$ Chalifoux N,${ }^{1}$ Whelan $\mathrm{J},{ }^{4}$ Meyer N,${ }^{5}$ Slensky K, ${ }^{1}$ Cleroux A, ${ }^{1}$ Weiss SR, ${ }^{4}$ O'Doherty $\mathrm{U},{ }^{2}$ and Rankin SC. ${ }^{3}$

1. Department of Clinical Sciences and Advanced Medicine, School of Veterinary Medicine, University of Pennsylvania.

2. Department of Laboratory Medicine and Therapeutic Pathology, Perelman School of Medicine, University of Pennsylvania.

3. Department of Pathobiology, School of Veterinary Medicine, University of Pennsylvania.

4. Department of Microbiology, Perelman School of Medicine, University of Pennsylvania.

5. Department of Pulmonary and Critical Care Medicine, Perelman School of Medicine, University of Pennsylvania.

*Address correspondence to mlennon@vet.upenn.edu

\begin{abstract}
SARS-CoV-2 infects a range of host species. However, the susceptibility of companion animals to SARS-CoV-2 and their potential ability to transmit the virus to humans remains unclear. Here, we present a detailed clinical description of an immunosuppressed dog that was infected with SARS-CoV-2. The dog had severe gastrointestinal $(\mathrm{Gl})$ clinical signs, coagulopathy, elevated hepatic transaminases, and met canine systemic inflammatory response syndrome criteria, without respiratory clinical signs, mirroring a subset of humans with GI-restricted COVID-19. Viral sequencing demonstrated divergence from other reported sequences, based on phylogenetic analysis. The dog shed high levels of virus for a prolonged time period with positive virus isolation. The dog's immunosuppressed state may have increased both susceptibility to infection and disease progression. Together, our findings suggest that certain individual companion animals may be at higher risk for severe SARS-CoV-2 infection, COVID-19-like disease, and high viral shedding, which may pose a transmission risk to humans.
\end{abstract}

\section{Introduction}

Severe acute respiratory syndrome-coronavirus-2 (SARS-CoV-2) is a zoonotic infection that has resulted in a worldwide pandemic, causing coronavirus disease 2019 (COVID-19). This virus infects a wide range of host species, yet little is known about the pathogenesis and clinical course in animals. Companion animals, such as dogs and 
cats, reside with SARS-CoV-2 infected owners, but despite severely limited systematic testing of companion animals to date, dogs are assumed to be resistant to infection based on experimental studies with young, healthy laboratory-bred animals. ${ }^{1,2}$ It is not known if certain conditions, such as advanced age, concurrent illness, or immunosuppressive medications increase the susceptibility of companion animals to SARS-CoV-2 as it does in humans. ${ }^{3-5}$ Naturally-exposed dogs and cats have tested positive for SARS-CoV-2, and some have had positive virus isolation, but most welldescribed cases have had minimal to no clinical signs, and many cases had low-level virus shedding. ${ }^{6,7}$ Prior to this report, the longest reported duration of shedding and detection of virus in a dog was 13 days. ${ }^{6}$ Moreover, while most affected companion animals were asymptomatic, certain individual animals did develop severe disease, yet very little clinical information has been published about these cases. To date, no published studies have described a clinical case of a dog with SARS-CoV-2 illness and with documented infection. A recent study investigating a small number of dogs and cats living with COVID-positive humans demonstrated that $3 / 17(17.6 \%)$ cats and $3 / 59(5.1 \%)$ dogs had a positive qPCR test.. ${ }^{7}$ Virus neutralizing antibody responses were more common: $7 / 16(43.8 \%)$ cats and $7 / 59(11.9 \%)$ dogs had a positive virus neutralization titer. SARS-CoV-2 was recovered by virus isolation in one cat, providing additional natural context to the experimental studies that have demonstrated that housecats can transmit SARS-CoV-2., 1, 8

Evidence has demonstrated that natural interspecies transmission events can pose a significant human health risk. In farmed mink, SARS-CoV-2 spillover from humans resulted in productive infections in mink. Mink-to-mink transmission was then documented on multiple farms worldwide, and ultimately resulted in a mutation in the spike protein sequence that posed a concern for vaccine efficacy. ${ }^{9-11}$ Multiple events of mink-to-human transmission then followed, and the virus retained the ability to efficiently spread between humans. ${ }^{10}$ The current report describes an immunosuppressed dog with severe SARS-CoV-2 infection, prolonged virus shedding with positive isolation, and clinical signs that mirrored human COVID-19 isolated to the GI tract, with SIRS, coagulopathy, and elevated hepatic transaminases. Together, these data demonstrate the importance of spillover infections in companion animals and emphasize that interspecies transmission are a public health risk. ${ }^{9,10}$

\section{Results}

On the evening of 13 December 2020 (Day -2), a 7-year-old spayed female mixed breed dog was presented to the veterinary hospital's emergency service with a 2day history of hematochezia, extreme lethargy, and one episode of vomiting. The dog's owner had developed symptoms of COVID-19 on 11 December 2020, approximately 36 hours before the onset of the dog's clinical signs, and tested positive for SARS-CoV-2 via a rapid antigen test on 13 December 2020. The dog's owner hand-fed the dog daily, including in the days leading up to the dog's presentation to the hospital. The spouse of the dog owner became symptomatic for COVID-19 on 14 December 2020. 


\section{Prior Diagnosis and Workup of Immune Mediated Hemolytic Anemia}

The dog had a history of immune mediated hemolytic anemia (IMHA) diagnosed four months prior. The dog was treated with immunosuppressive doses of prednisone (1.4 mg/kg/day), as well as mycophenolate and clopidogrel, and the IMHA responded well to treatment. Bloodwork was monitored at recommended intervals, and the medications were tapered by the attending veterinarian over the following months. The most recent recheck before the current presentation was on 1 December 2020, 12 days prior to the presentation to the emergency service. At that recheck, the hematocrit and reticulocyte count were normal. All white blood cell parameters were normal except for mild lymphopenia $\left(0.63 \times 10^{3} / \mu \mathrm{l}\right.$ (reference range: $\left.0.9-4.7 \times 10^{3} / \mu \mathrm{l}\right)$ ). Her prednisone was tapered to $0.42 \mathrm{mg} / \mathrm{kg} / \mathrm{day}(0.67 \mathrm{mg} / \mathrm{kg} /$ day based on lean body weight).

\section{Presentation and Diagnosis of SARS-CoV-2 Infection}

On presentation to the emergency service, the dog had depressed mentation and was febrile with a body temperature of $105.4^{\circ} \mathrm{F}$. Tachycardia, tachypnea, and increased respiratory effort were observed on physical examination. Frank blood was noted on rectal examination, and the dog was dribbling urine and demonstrated signs of discomfort on abdominal palpation. The dog was assessed to be $5-8 \%$ dehydrated. Hypersalivation and pale pink mucous membranes were noted. Notably, the dog's vital signs met the criteria for canine systemic inflammatory response syndrome (SIRS) and she was hypotensive. A venous blood gas demonstrated a metabolic acidosis with elevated lactate concentration and electrolyte dysregulation (mild hyponatremia, hypochloremia, hypokalemia). Stabilization was achieved with fluid boluses, electrolyte monitoring via venous blood gas, and routine supportive care.

Because the dog met canine SIRS criteria, enrofloxacin and metronidazole were administered due to concern for possible sepsis. Beta-lactam and cephalosporin antimicrobials were avoided initially due to the patient's history of IMHA. During administration of enrofloxacin, the dog developed paroxysmal supraventricular tachycardia that was non-responsive to vagal maneuver. Diltiazem was administered; first- and second-degree atrioventricular block then developed, which resolved after several hours. No further arrhythmias were noted during hospitalization.

A chemistry panel demonstrated moderate elevation of liver transaminases (increased alanine aminotransferase (ALT) (727 U/L, reference range 16-91 U/L), aspartate aminotransferase (AST) (168 U/L; reference range 23-65 U/L)), and alkaline phosphatase (ALP) (308 U/L; reference range 20-155 U/L)) with electrolyte abnormalities as previously noted on venous blood gas. Thoracic radiographs were performed which revealed a mild generalized bronchial pattern, as previously noted on thoracic radiographs performed 4 months earlier as well as possible new sternal lymphadenopathy. Thoracic radiographs were not repeated during the remainder of the hospitalization, as no additional respiratory clinical signs were noted. The dog's oxygen saturation as measured by pulse oximetry was normal throughout the dog's hospitalization.

The morning following presentation (14 December 2020, Day -1)), a complete blood count $(\mathrm{CBC})$ showed a new anemia with a hematocrit of $32.5 \%$ (reference range 
$41-58 \%$ ), total leukopenia (characterized by a neutropenia of $1.93 \times 10^{3} / \mu \mathrm{l}$ (reference range 2.7-9.4 $\left.\times 10^{3} / \mu \mathrm{l}\right)$, with mild toxic change and occasional band neutrophils $(0.3 \times$ $10^{3} / \mu \mathrm{l}$; reference range $\left.0-0.1 \times 10^{3} / \mu \mathrm{l}\right)$, severe lymphopenia $\left(0.38 \times 10^{3} / \mu \mathrm{l}\right.$; reference range $\left.0.9-4.7 \times 10^{3} / \mu \mathrm{l}\right)$, and thrombocytopenia of $\left(162 \times 10^{3} / \mu \mathrm{l}\right.$; reference range $186-545$ $\left.\times 10^{3} / \mu \mathrm{l}\right)$ with large platelets noted. Presence of moderate rouleaux was noted. The neutrophil-to-lymphocyte ratio (NLR) was 5.1. The chemistry panel revealed persistent hyponatremia, hypokalemia, and hypochloremia with normal anion gap. The ALT had progressively increased (917 U/L, reference range 16-91 U/L) with normal bilirubin and cholesterol. Albumin and globulins were normal. Urinalysis revealed a urine specific gravity of 1.010 (post-fluid therapy), $\mathrm{pH} \mathrm{8,} \mathrm{and} \mathrm{an} \mathrm{inactive} \mathrm{sediment.} \mathrm{A} \mathrm{urine} \mathrm{culture} \mathrm{was}$ performed and was negative. There was evidence of a coagulopathy: D-dimer was elevated at 1.7 (reference range: $0-0.2 \mathrm{ug} / \mathrm{ml}$ ) and partial thromboplastin time (PTT) was minimally prolonged at 16.6 (reference range: 10.7-16.4). Prothrombin time (PT) was within the reference interval. An ammonia concentration was normal. The dog remained febrile, and a decision was made to change antibiotic therapy to clindamycin and ceftazidime after 24 hours.

An abdominal ultrasound was performed that showed nonspecific changes consistent with enteritis or colitis. A fecal examination revealed no intestinal parasites. Fecal cultures for Salmonella spp. and Campylobacter spp. were negative. A PCR panel for gastrointestinal organisms was submitted to a commercial diagnostic laboratory and was negative for all organisms included (canine enteric coronavirus, Campylobacter jejuni/coli, Cryptosporidium spp., Salmonella spp. Giardia spp. Clostridioides difficile toxins $\mathrm{A}$ and $\mathrm{B}$, Clostridium perfringens enterotoxin, and canine parvovirus). On 14 December 2020 (Day -1), fecal and saliva specimens were tested for SARS-CoV-2 by real time reverse transcriptase polymerase chain reaction (RTPCR) using the Centers for Disease Control (CDC) 2019 Novel Coronavirus (2019nCoV) RT-PCR Diagnostic Panel. Both specimens were negative.

On 15 December 2020 (Day 0), the dog's fever resolved, she began eating, and was able to rise and go for a short walk. Diarrhea continued but was without hematochezia. a CBC and serum chemistry panel were again performed and demonstrated persistent but improving anemia, persistent lymphopenia, and a normal neutrophil count with mild toxic change and no band neutrophils. The chemistry panel demonstrated continuing elevation in liver enzymes with a predominantly hepatocellular pattern (ALT $917 \mathrm{U} / \mathrm{L}$ ). Testing for SARS-CoV-2 by RT-PCR was again performed, and this time all specimens (nasopharyngeal swab, saliva, and feces) were positive. The nasopharyngeal swab specimen had cycle threshold (CT) values of 16.7 and 17.1 for the N1 and N2 targets, respectively. The CT values for saliva were 28.1/27.6, and the 37.1/36.8 for feces (Figure 1). 


\section{Cycle Threshold Value, SARS-CoV-2 qPCR}

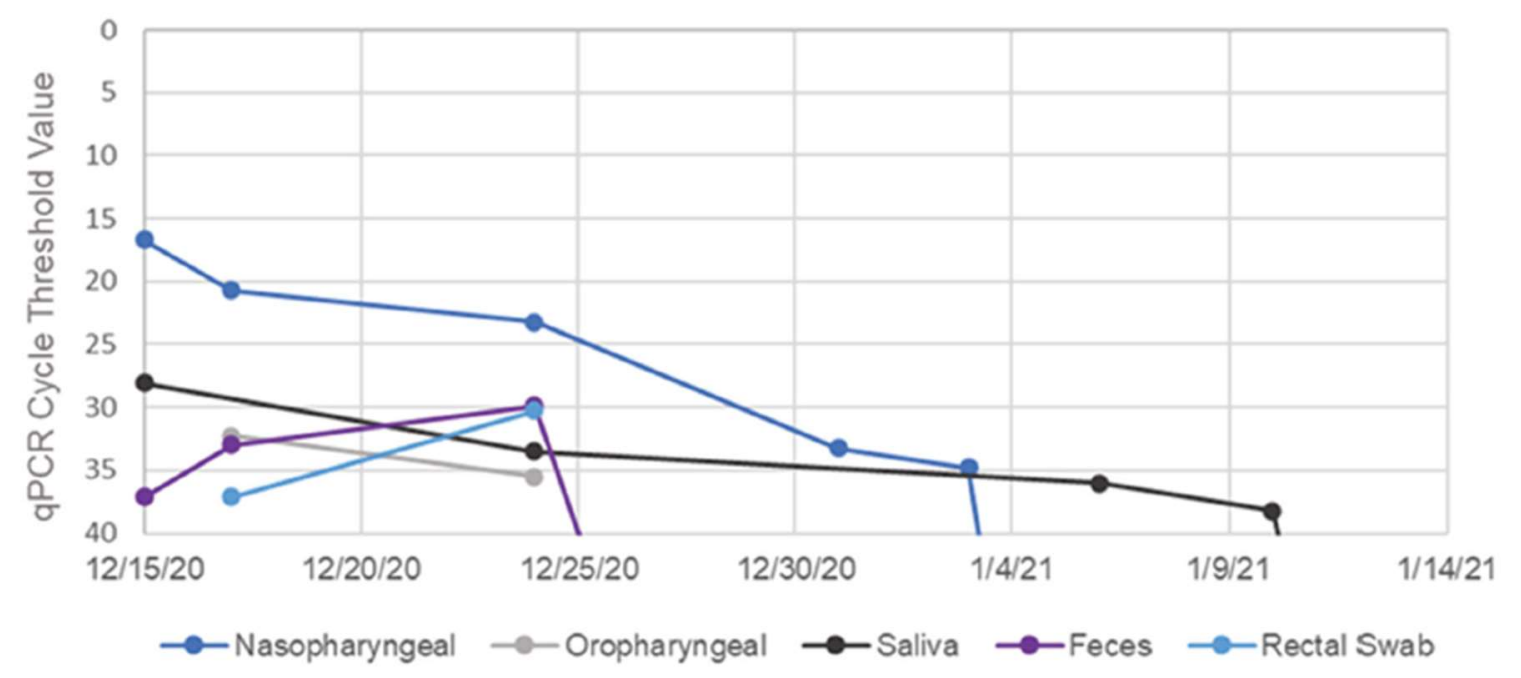

Figure 1: Longitudinal qPCR Cycle threshold (CT) values by body site. Longitudinal sampling revealed highest viral load in the nasopharynx, but longest persistence of SARS-CoV-2 in saliva.

To comply with mandates for this reportable disease, aliquots of the original specimens were sent to the National Veterinary Services Laboratory ((NVSL), Ames, IA) and were confirmed to be positive with a similar CT values: nasopharyngeal swab $18.4 / 19$, saliva 31/30.8; the virus was not detected in the fecal sample. Virus isolation performed at NVSL with the nasopharyngeal swab collected on 17 December 2020 was reported as positive on January 15, 2021. Virus sequencing was performed at NVSL, but the results have not been made available at this time. Virus neutralization testing on a serum specimen collected on 15 December 2020 was negative for antibody neutralization.

The dog was hospitalized until 17 December 2020 for continuing supportive care, and monitored bloodwork showed gradual improvement. Specimens were again collected for SARS-CoV-2 RT-PCR on 17 December 2020 (Day 2) and all specimens again tested positive. The nasopharyngeal swab had CT values of 20.7/19.5, an oropharyngeal swab was tested and the CT values were 32.3/31.2, the CT values from feces were $33 / 35.3$, and it should be noted that this is lower than the previous fecal specimen tested on 15 December 2020, indicating a higher viral load. The dog was transitioned to oral medications and was discharged. All individuals residing in the house had already been diagnosed with COVID-19, and the dog was isolated along with the owners. Following discharge, the dog continued to improve at home and the owner noted no remaining clinical signs.

A recheck examination was performed on 24 December 2020 (Day 9) and the dog's physical examination was unremarkable. A CBC and serum chemistry panel were performed, which showed a regenerative resolving anemia, mild mature neutrophilia, 
and a normal lymphocyte count. The serum chemistry panel showed a resolving hepatopathy and persistent mild hypochloremia. Although this dog had been treated with long-term prednisone therapy for IMHA, a SARS-CoV-2 virus neutralization assay performed on serum collected on 24 December 2020 was positive at a titer of 1:8.

Specimens collected for SARS CoV-2 PCR on 24 December 2020 (Day 9) were tested at the university microbiology laboratory but were not submitted to NVSL because the dog had already been confirmed as infected. A nasopharyngeal swab, saliva and a rectal swab were positive with CT values of 23.2/22.9, 33.5/34.9, and 29.9/31.2 respectively. On 25 December 2020 (Day 10), a naturally voided fecal specimen had a CT value of 29.9/31.2. Remarkably, the rectal swab and fecal CT values showed increasing intestinal shedding of virus, while nasopharyngeal and saliva shedding was declining. Repeated longitudinal specimens were collected for SARSCoV-2 PCR (Figure 1), and at least one specimen and gene target had persistent detection of SARS-CoV-2 through 10 January 2021 (Day 26). On 14 January 2021 (Day $30)$, all specimens collected tested negative, and specimens collected at 3 time points afterwards, through 23 February 2021 (Day 70), remained negative. As virus can typically be isolated from human specimens with CT values $<24$, the CT values in the nasopharyngeal specimens demonstrate high levels of virus shedding for at least 9 days. . $6,12,13$ The dog tested positive for SARS-CoV-2 for 26 days after the initial diagnosis, which is the longest period of persistent viral shedding reported in a dog. This long duration of shedding is consistent with what has been reported in humans and cats, but has not been previously demonstrated in dogs. ${ }^{7,14-16}$

The dog recovered completely from illness, and the prednisone was tapered and then discontinued. A recheck examination 1 month after discontinuing the medication revealed normalization of all bloodwork parameters at the last recheck on 9 March 2021.

Repeated serum virus neutralization assays were collected on 6 January (Day 22), 28 January (Day 44), and 23 February 2021 (Day 70), and were tested at NVSL. The three serum samples tested showed titers of 16,32 , and 32 , respectively. These results suggest that the dog did mount a delayed antibody response that was weaker than expected given the severity of illness. A multiplex cytokine assay was performed on longitudinal specimens from the dog, and revealed marked immune activation that resolved with time, characterized by a strong CXCL8 response, with a spike in IL-15 during the recovery phase (Figure 2). TNF was undetectable, and IL-6 and IFN-Y were not markedly elevated. Similarly, in humans, the cytokine response to SARS-CoV-2 is characterized by elevations in CXCL8 and IL-15, amongst other cytokines. ${ }^{17}$ While TNF and IL-6 are elevated in most humans with pulmonary COVID-19, ${ }^{18}$ these cytokines are not uniformly elevated in all patients. Furthermore, some evidence suggests that cytokine induction can be less powerfully induced in gastrointestinal than pulmonary COVID-19. ${ }^{19}$ 

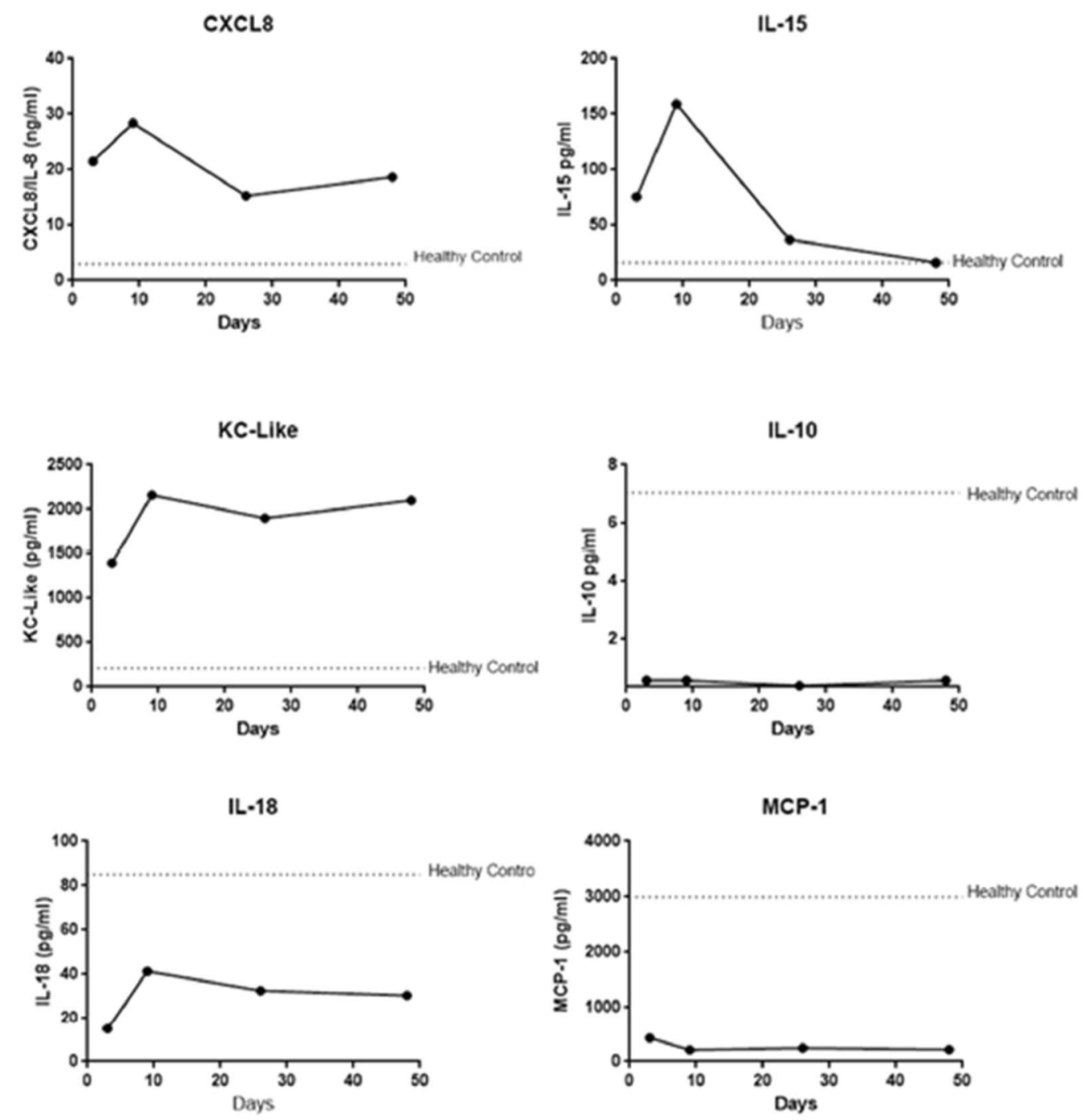

\section{Figure 2: Longitudinal serum cytokine concentrations during infection and recovery.}

Viral RNA sequencing was performed, and revealed that the dog was infected with the SARS-CoV-2 B.1.509 variant, consisting of the D164G spike protein substitution that is the dominant variant commonly present in the US. The SARS-CoV-2 genome from the dog did not cluster with other sequences in a phylogenetic tree (Figure 3). SARS-CoV-2 consensus sequences from Pennsylvania were used for comparison (CDC, deposited in GISAID). Similar results were obtained with unpublished sequences from the greater Philadelphia area, and B.1.509 has not been previously identified in this dataset (Rick Bushman, personal communications). ${ }^{20}$ 
Tree scale: 0.001

Figure 3: SARS-CoV-2 genome from the dog did not cluster with other sequences from Pennsylvania in a phylogenetic tree.

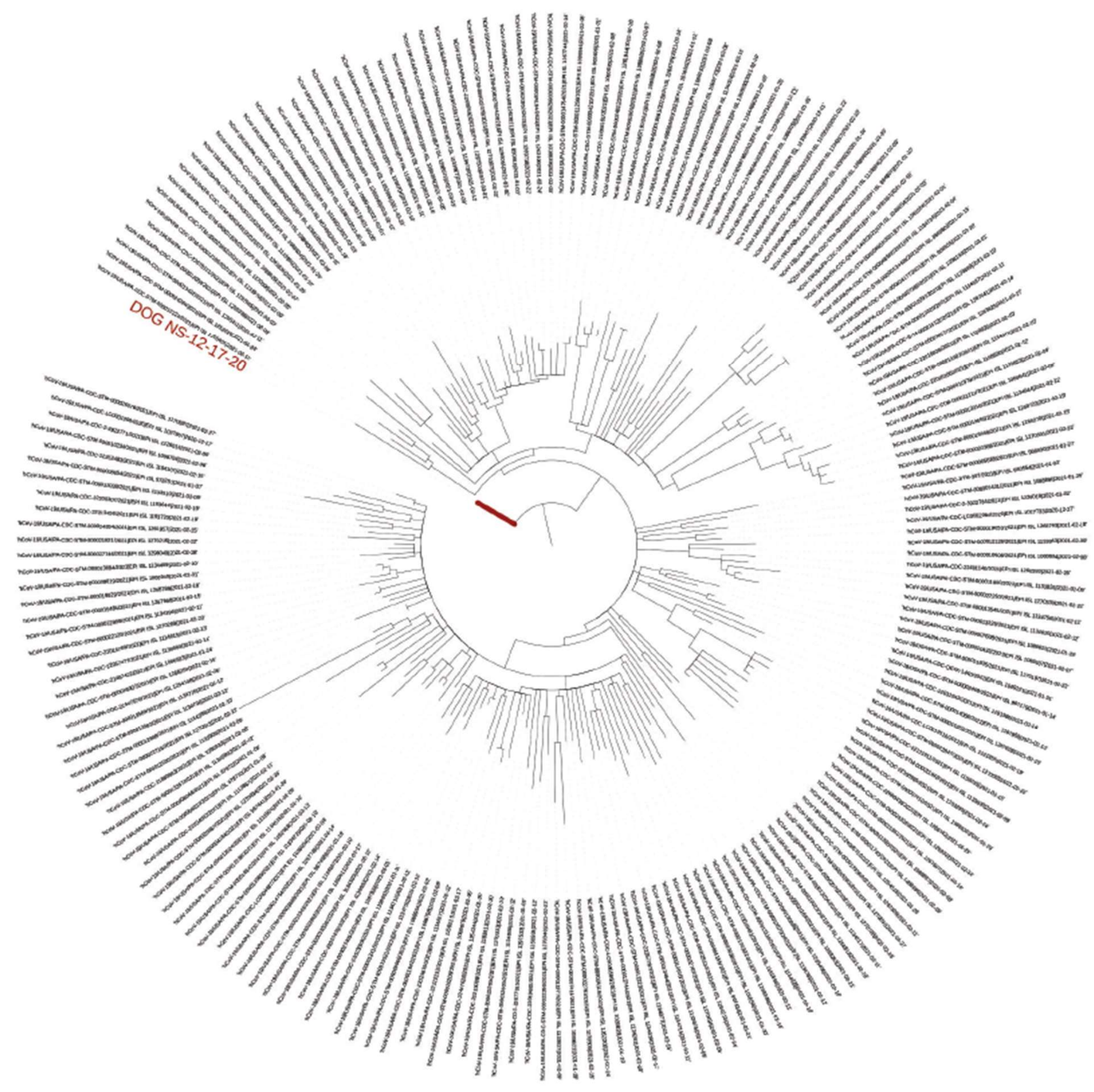




\section{Discussion}

Here we present the first detailed case report of an immunosuppressed dog with clinical illness and SARS-CoV-2 infection that mirrors human COVID-19 with symptoms restricted to the gastrointestinal tract. Although COVID sepsis has not been previously reported in dogs, on presentation, this dog met canine SIRS criteria, and thus met sepsis criteria given the dog's SARS-CoV-2 infection, and demonstrated coagulopathy with elevated hepatic transaminases. 21,22 The dog shed high levels of virus for a prolonged time period and SARS-CoV-2 was isolated from a nasopharyngeal swab specimen collected on 17 December 2020. It is possible that the immune suppressed state of this dog contributed to its susceptibility to infection and the development of severe disease. Furthermore, the virus was highly divergent from all other publicly available SARS-CoV-2 sequences as well as unpublished sequences from Philadelphia, suggesting that the prolonged course and immune suppressed state of the dog could have contributed to viral mutation, as has been previously suggested in humans. ${ }^{23,24}$ In support, this dog developed a weak antibody response as measured by virus neutralization assay, only reaching a titer of 1:32 after more than 6 weeks. In contrast, asymptomatic dogs in a recent report demonstrated higher titers, up to $1: 128 .^{7}$

Together, our findings highlight individual variability in susceptibility to SARSCoV-2 in dogs, as is observed in humans, and suggest that certain subsets of companion animals may be at higher risk for SARS-CoV-2 infection, and subsequently may pose a greater risk for transmission to humans. Further study to determine risk factors for SARS-CoV-2 infection in companion animals is necessary.

The dog in this report presented to the hospital with primarily severe gastrointestinal clinical signs. This report demonstrates that clinical signs of SARS-CoV2 can be restricted to the gastrointestinal tract in dogs, which may have resulted in under-reporting of this virus in dogs, especially given the restrictions in place for companion animal testing for SARS-CoV-2. Gastrointestinal symptoms are relatively common in human patients with COVID-19, and in approximately $2 \%$ of human cases, symptoms are restricted to the gastrointestinal tract. ${ }^{25}$ Gastrointestinal symptoms can be the earliest or the primary manifestation of human disease. ${ }^{21,22,26-28}$ In humans, SARS-CoV-2 infection can cause gastrointestinal bleeding, ulceration, and ischemia from thromboembolism. ${ }^{29-31}$

Because the dog fit SIRS criteria on presentation to the hospital, the attending clinician chose to immediately begin treatment with antibiotics due to suspicion of bacterial sepsis, and blood cultures were not collected at the time of admission. Concurrent bacterial sepsis and COVID-19 have been previously reported in humans, with up to $7 \%$ of hospitalized COVID-19 patients having bacterial co-infections. ${ }^{32,33}$

Remarkably, the dog did not have evidence of significant respiratory disease. Although the dog's respiratory rate was elevated on presentation, it normalized within a few hours of supportive care, as did her heart rate and blood pressure. Thoracic radiographs revealed possible sternal lymphadenopathy, but no evidence of pulmonary parenchymal disease. In humans, mediastinal lymphadenopathy can occasionally be seen with COVID-19. ${ }^{34}$ In addition, this dog had evidence of liver injury, which is commonly reported in human COVID-19, and can occasionally be severe. ${ }^{5,35}$ The 
mechanism of liver injury remains unclear, but may be due direct infection of liver cells with SARS-CoV-2 and systemic inflammation. ${ }^{36}$

Consistent with previously reported data in humans ${ }^{37}$ in whom SARS-CoV-2 RNA levels peak in the nasopharynx 2-6 days after the onset of symptoms, ${ }^{38-40}$ the dog tested negative for SARS-CoV-2 on the first day of sampling of saliva and feces. However, as SARS-CoV-2 infection was suspected and saliva and fecal specimens are less sensitive than nasopharyngeal swabs for the detection of this virus, ${ }^{39,41}$ the dog was re-sampled on 15 December 2020 and a more sensitive nasopharyngeal swab was collected in addition to saliva and fecal specimens, and all were positive. As in humans, the nasopharyngeal swab specimen displayed the highest sensitivity for SARS-CoV-2 detection in this dog. ${ }^{42}$ We successfully detected SARS-CoV-2 in saliva specimens in a dog, suggesting that this less invasive nature of specimen collection may be more practical for some applications. A recent study compared paired nasopharyngeal and saliva sampling and found that 2 individuals had persistent viral detection in saliva despite a negative nasopharyngeal swab, similar to the dog in this report. ${ }^{43}$

Previous studies in young, healthy dogs exposed to SARS-CoV-2 in experimental settings have demonstrated limited susceptibility to SARS-CoV-2, with seroconversion but little viral shedding. ${ }^{1,2}$ Reports of naturally infected dogs have identified some cases in which SARS-CoV-2 can be detected, indicating that studies in laboratory dogs are not representative of the entire canine population, but generally dogs have no clinical signs or mild signs. ${ }^{6,7,44,45}$ The dog in this case report was being treated with prednisone for previously diagnosed IMHA, which may have contributed to the high viral load and atypical clinical signs observed. Solid organ transplant patients, who are receiving immunosuppressive medications, also have increased severity of COVID-19. ${ }^{3}$ Similarly, in patients with inflammatory bowel disease, corticosteroid treatment, but not TNF antagonist treatment, is associated with worse outcomes in COVID-19. ${ }^{4}$ Further study of dogs that are exposed to SARS-CoV-2 and are receiving corticosteroids and other immunosuppressant therapy is warranted, given that any enhanced viral shedding could increase zoonotic risk.

Interspecies transmission events are a central component of SARS-CoV-2 evolution and emergence in humans. The worldwide distribution of SARS-CoV-2, with intense infection of the United States population, has brought SARS-CoV-2 into close contact with millions of companion animals that share human households. ${ }^{46}$ In some areas with widespread human infection, community prevalence of SARS-CoV-2 antibodies reported in companion animals is relatively high. ${ }^{47,48}$ Indeed, recent published and preprint manuscripts that studied dogs and cats living with COVIDpositive humans demonstrated that spillover infection of companion animals is much more common than previously realized. ${ }^{7,49}$ Viable virus was recovered from one cat, providing additional natural context to the experimental studies that have demonstrated that housecats can transmit SARS-CoV-2. ${ }^{1,8}$ Interspecies transmission events pose a risk for SARS-CoV-2 mutation since they impose unique selection pressures on the virus and an in vitro study recently demonstrated that transmission in cats imposes selection pressure on the spike protein sequence required for viral entry. ${ }^{50}$ 
This case provides new data on the magnitude of potential virus shedding by dogs. To the authors' knowledge, the PCR CT values documented in this dog is consistent with the highest observed magnitude of virus shedding of SARS-CoV-2 in a dog, and the longitudinal analysis is consistent with the longest duration of virus shedding reported in the literature to date. One previous case documented prolonged shedding of SARS-CoV-2 in a dog, with viable virus recovered, although viral shedding in the previously reported case was dramatically lower and of shorter duration (13 days) ${ }^{6}$ than the dog in the current report, which tested positive by PCR for 27 days, including 9 days with a high level of virus shedding (CT values less than 24). Importantly, the dog in the previous report showed no clinical signs of illness. In humans, CT values less than 24 are typically considered to have the highest transmission risk. ${ }^{12,13}$ The SARS-CoV-2 virus was isolated from this dog and that underscores the presence of live, viable virus in large numbers.

Very early screening of dog and cat specimens from mid-February 2020 to midApril 2020 by a commercial veterinary diagnostic laboratory did not support companion animal infection with SARS-CoV-2. ${ }^{51}$ However, as this report illustrates, there is still much to learn about SARS-CoV-2 infection of companion animal species. It is possible that there are factors, such breed, age or immunosuppression, or as-yet-undiscovered genetic factors or comorbidities that could increase the susceptibility of some companion animals to SARS-CoV-2.

However, there are currently significant barriers in place that severely limit companion animal testing. Clinical testing is reserved for cases in which more common diseases have been ruled out, yet many owners do not have the financial resources to pay out-of-pocket for extensive diagnostic testing. Therefore, it is possible that cases of SARS-CoV-2 infection may have been missed in companion animals, particularly in animals that have clinical signs of disease outside of the respiratory tract. This case highlights the fact that there are many gaps in our knowledge about manifestations of SARS-CoV-2 infection in companion animals. It also indicates the need for more widespread testing of animals that have clinical signs in general.

In summary, this report describes an immunosuppressed dog with clinical infection of the gastrointestinal tract with SARS-CoV-2, high viral shedding and positive virus isolation. The unique and severe gastrointestinal presentation highlights the need for further investigation of the full spectrum of COVID-19 in companion animals, including the associated human health risks.

\section{Materials and Methods}

All procedures presented in this manuscript were either performed as part of routine medical care or were approved under an approved IACUC and privately owned animal protocol (POAP) at the University of Pennsylvania. Owner consent was obtained for all procedures. Consent was obtained from the state animal health officials to collect specimens from the dog for SARS-CoV-2 testing, and for submission of "non-negative" specimens to NVSL for confirmation. The positive status of this animal was reported to the World Organization for Animal Health on 31 December 2020 by Dr. Mark Davidson 
Associate Administrator, USDA-APHIS, United States Department of Agriculture, Washington, United States of America. Clinical complete blood count and serum chemistry profiles were performed at the university veterinary diagnostic laboratory using equipment approved by a Clinical Pathologist board certified by the American College of Veterinary Pathologists. All thoracic and abdominal imaging studies were performed and interpreted by radiologists board certified by the American College of Veterinary Radiology.

\section{SARS-CoV-2 Clinical Testing}

RNA was extracted from specimens using a QIAamp Viral RNA Mini (Qiagen, Germantown, MD). Testing for SARS-CoV-2 was performed at the university microbiology laboratory using the CDC 2019 Novel Coronavirus (2019-nCoV) RealTime Reverse Transcriptase (RT)-PCR Diagnostic Panel (IDT, Coralville, IA). The university microbiology laboratory is a member laboratory of the Food and Drug Administration (FDA) Veterinary Laboratory Investigation and Response Network (VetLIRN). As part of this network, the university microbiology laboratory completed an Inter-Laboratory Comparison Exercise (ICE) of SARS-CoV-2 Molecular Detection Assays Being Used by Veterinary Diagnostic Laboratories in August 2020.

\section{Virus neutralization assay}

A virus neutralization assay was performed at the National Veterinary Services Laboratory in accordance with their standard operating procedures, using two-fold serially diluted sera (final dilution beginning at 1:8).

\section{Virus Isolation}

Virus isolation was performed according to standard techniques at the National Veterinary Services Laboratory, Ames, IA, as well as the laboratory of SRW. In the SRW laboratory, virus from clinical samples was isolated as previously described. ${ }^{20}$ Samples were incubated 1:1 in DMEM (Gibco catalog no. 11965) with 2\% FBS, $200 \mathrm{U}$ penicillin, $200 \mu \mathrm{g} / \mathrm{ml}$ streptomycin, $50 \mu \mathrm{g} / \mathrm{ml}$ gentamicin, and $2.5 \mu \mathrm{g} / \mathrm{ml}$ amphotericin B for $1 \mathrm{~h}$ at $37^{\circ} \mathrm{C}$. Nasopharyngeal swabs used for collection were mixed with DMEM with $2 \%$ FBS, $200 \mathrm{U}$ penicillin, $200 \mu \mathrm{g} / \mathrm{ml}$ streptomycin, $50 \mu \mathrm{g} / \mathrm{ml}$ gentamicin, and $2.5 \mu \mathrm{g} / \mathrm{ml}$ amphotericin B in a 24-well plate before incubation for $1 \mathrm{~h}$ at $37^{\circ} \mathrm{C}$. Samples were then inoculated onto A549ACE2 cells or VERO-TMPRSS2 cells in 24-well plates and incubated for $1 \mathrm{~h}$ at $37^{\circ} \mathrm{C}$. After $1 \mathrm{~h}$, inoculum was removed and $0.5 \mathrm{~mL}$ DMEM with $2 \% \mathrm{FBS}, 200 \mathrm{U}$ penicillin, $200 \mu \mathrm{g} / \mathrm{ml}$ streptomycin, $50 \mu \mathrm{g} / \mathrm{ml}$ gentamicin, and $2.5 \mu \mathrm{g} / \mathrm{ml}$ amphotericin B was added to each VERO-TMPRSS2 well, or RPMI 1640 (Gibco catalog no. 11875) with 2\% FBS, $200 \mathrm{U}$ penicillin, $200 \mu \mathrm{g} / \mathrm{ml}$ streptomycin, $50 \mu \mathrm{g} / \mathrm{ml}$ gentamicin, and $2.5 \mu \mathrm{g} / \mathrm{ml}$ amphotericin B was added to each A549ACE2 well. Three to 4 days post-infection, supernatants were harvested and $300 \mu \mathrm{l}$ was used to inoculate A549ACE2 cells in 6-well plates. Forty-eight hours postinfection, the supernatants were collected, the cells were lysed using RLT Plus lysis buffer, and the RNA was extracted using the RNeasy Plus Mini Kit (Qiagen). 


\section{Multiplex Cytokine Analysis}

Quantitation of GM-CSF, IFN-y, IL-2, IL-6, IL-7, IL-8, IL-15, IP-10, KC-like, IL-10, IL-18, MCP-1, and TNF- $\alpha$ was performed by the Translational Core Laboratory at the Children's Hospital of Philadelphia using a commercially available canine-specific assay (BD Cytometric Bead Array Kit (BD Biosciences, San Jose, CA, USA). The assay was performed using a solid phase protein immunoassay that uses spectrally encoded antibody-conjugated beads as the solid support (Luminex). Following the manufacturer instructions, standards and samples are added to wells containing antibody-conjugated beads, and results are obtained by monitoring the spectral properties of the capture beads while simultaneously measuring the quantity of associated fluorophore. Standard curves and quality control samples were performed on each plate. Coefficient of variation (\%) was accepted if $<20 \%$.

\section{SARS-CoV-2 genome amplification and sequencing}

SARS-CoV-2 genomes were amplified using the ARTIC protocol after RNA isolation by Trizol. ${ }^{52}$ Briefly, RT-PCR using random primers with SuperScriplll was performed at $55^{\circ} \mathrm{C}$ for 60 minutes followed by 10 minutes at $70{ }^{\circ} \mathrm{C}$. The products were then amplified by PCR using the ARTIC primers V3 pools 1 and 2 and NEB Q5 Hot Start High-Fidelity polymerase. The PCR cycling conditions were $98^{\circ} \mathrm{C}$ for 30 seconds; then $95^{\circ} \mathrm{C}$ for 15 seconds, $63{ }^{\circ} \mathrm{C}$ for $5 \mathrm{~min}$ for 35 cycles. PCR amplicons were purified using the DNA Clean \& Concentrator kit (Zymogen). DNA concentration was measured using Qubit Fluorometric Quantification. Finally, the Nextera library preparation kit (Illumina) was used to sequenced the purified amplicons on a MiniSeq System using a Mid-output flow cell (Illumina).

\section{Sequence assembly and analysis.}

Paired reads were trimmed in Geneious using "BBDuk" plugin, discarding reads from the adaptor, and then merged using Geneious. The reads were trimmed again to remove nucleotide calls with a quality rating under 30 . The reads were then mapped using BBMap to as reference "SARS coronavirus 2 isolate Wuhan-Hu-1"

(NC_045512.2), to extract those that aligned to the reference sequence. Consensus sequences were generated through de novo assembly of the extracted reads using "Spades" Geneious plugin. We identified nucleotide changes in the dog consensus sequence compared to the Wuhan reference. We next determined which of these mutations were already reported in the GISAID database. ${ }^{53}$ We then generated a phylogenetic tree using 200 sequences from the Pennsylvania region that were recently deposited in GISAID. ${ }^{53}$

Viral lineage was determined using PANGOLIN software. A phylogenetic tree was constructed using SARS-CoV-2 sequences isolated from individuals in Pennsylvania (deposited by the CDC in GISAID); 50\% were from the Philadelphia region (personal communication Duncan MacCannell). Sequences with $>99.5 \%$ similarity were clustered using CD-HIT-EST, and a representative sequence from each cluster was included in the phylogeny. The SARS-CoV-2 sequence from the immunocompromised dog on 12/17/20 and the representative Pennsylvania sequences were then aligned with MAFFT. A maximum-likelihood phylogenetic tree was generated 
with Geneious software using the PhyML 3.3.20180621 plugin and the Jukes-Cantor69 substitution model. The tree was visualized with the iTOL webpage.

Data availability: All relevant data are available from the authors upon request.

Acknowledgements: The authors would like to thank Dr. Rick Bushman for advice regarding viral sequencing and for comparing the results to unpublished Philadelphia sequences in his laboratory. Sam Weissman generously contributed to sequence analysis. Dr. Lennon would like to acknowledge Dr. De'Broski Herbert for mentorship advice regarding this publication. The authors would like to acknowledge the owners of the dog for their motivation to generate new knowledge and their willingness to be available for follow up sampling. The authors would like to acknowledge Leslie King for manuscript editing and advice. The authors would like to acknowledge the National Veterinary Services Laboratory staff for confirming the RT-PCR results obtained in the university microbiology laboratory, virus isolation, and the virus neutralization assays.

This work was supported in part by the Institute for Translational Medicine and Therapeutics of the Perelman School of Medicine and the School of Veterinary Medicine at the University of Pennsylvania (Pilot Grant, co-Pls: Lennon/Weiss), the PennVet COVID Pilot Grant Program (PI: Lennon), and NIH R03OD026599 which provided salary support for Dr. Lennon.

University microbiology lab infrastructure support and funding for testing kits, supplies, and reagents were provided by the Food and Drug Administration Veterinary Laboratory Investigation and Response Network (VetLIRN), grant number U18FD006669 (PI: Rankin).

Research reported in this publication was supported by the National Center for Advancing Translational

Sciences of the National Institutes of Health under Award Number UL1TR001878. The content is solely the responsibility of the authors and does not necessarily represent the official views of the $\mathrm{NIH}$

Author contributions: EML conceptualized the project, wrote the manuscript, collected and processed specimens, and provided the funding. SR conceptualized the project, developed testing methodology, processed specimens, and edited the primary manuscript. NC and KS provided clinical care, initiated SARS-CoV-2 testing and provided feedback on the drafts. EB performed clinical testing and optimization. SC contributed to testing methodology, discussed results and helped design study method. $A C$ recruited patients for testing, conceptualized the project, and edited drafts. UOD supervised virus sequencing and analysis. AO performed viral genome sequencing and analysis. SW and JW performed viral isolation

All authors edited the manuscript.

Competing interests: The authors declare no competing interests. 
Materials \&Correspondence: Correspondence and material requests should be addressed to: mlennon@vet.upenn.edu

\section{References}

1. Shi J, Wen Z, Zhong G, et al. Susceptibility of ferrets, cats, dogs, and other domesticated animals to SARS-coronavirus 2. Science 2020:eabb7015.

2. Bosco-Lauth AM, Hartwig AE, Porter SM, et al. Experimental infection of domestic dogs and cats with SARS-CoV-2: Pathogenesis, transmission, and response to reexposure in cats. Proc Natl Acad Sci U S A 2020;117:2638226388.

3. Nair V, Jandovitz N, Hirsch JS, et al. An early experience on the effect of solid organ transplant status on hospitalized COVID-19 patients. Am J Transplant 2020.

4. Brenner EJ, Ungaro RC, Gearry RB, et al. Corticosteroids, But Not TNF Antagonists, Are Associated With Adverse COVID-19 Outcomes in Patients With Inflammatory Bowel Diseases: Results From an International Registry. Gastroenterology 2020;159:481-491.e3.

5. Chen N, Zhou M, Dong X, et al. Epidemiological and clinical characteristics of 99 cases of 2019 novel coronavirus pneumonia in Wuhan, China: a descriptive study. The Lancet 2020;395:507-513.

6. Sit THC, Brackman CJ, Ip SM, et al. Infection of dogs with SARS-CoV-2. Nature 2020.

7. Hamer SA, Pauvolid-Correa A, Zecca IB, et al. Natural SARS-CoV-2 infections, including virus isolation, among serially tested cats and dogs in households with confirmed human COVID-19 cases in Texas, USA. bioRxiv 2020:2020.12.08.416339.

8. Halfmann PJ, Hatta M, Chiba S, et al. Transmission of SARS-CoV-2 in Domestic Cats. New England Journal of Medicine 2020.

9. Lassaunière R FJ, Rasmussen $M$, Frische $A$, Strandh $C P$, Rasmussen TB, Botner A, Fomsgaard A. Working paper on SARS-CoV-2 spike mutations arising in Danish mink, their spread to humans and neutralization data. .

10. Oude Munnink BB, Sikkema RS, Nieuwenhuijse DF, et al. Transmission of SARS-CoV-2 on mink farms between humans and mink and back to humans. Science 2020:eabe5901.

11. Oreshkova N, Molenaar RJ, Vreman S, et al. SARS-CoV-2 infection in farmed minks, the Netherlands, April and May 2020. Eurosurveillance 2020;25:2001005.

12. Bullard J, Dust K, Funk D, et al. Predicting infectious SARS-CoV-2 from diagnostic samples. Clinical infectious diseases : an official publication of the Infectious Diseases Society of America 2020:ciaa638.

13. Rao SN, Manissero D, Steele VR, et al. A Narrative Systematic Review of the Clinical Utility of Cycle Threshold Values in the Context of COVID-19. Infectious diseases and therapy 2020:1-14.

14. Zhou B, She J, Wang Y, et al. Duration of Viral Shedding of Discharged Patients With Severe COVID-19. Clin Infect Dis 2020;71:2240-2242. 
15. Zhou F, Yu T, Du R, et al. Clinical course and risk factors for mortality of adult inpatients with COVID-19 in Wuhan, China: a retrospective cohort study. Lancet 2020;395:1054-1062.

16. van Kampen JJA, van de Vijver DAMC, Fraaij PLA, et al. Duration and key determinants of infectious virus shedding in hospitalized patients with coronavirus disease-2019 (COVID-19). Nature Communications 2021;12:267.

17. Huang C, Wang Y, Li X, et al. Clinical features of patients infected with 2019 novel coronavirus in Wuhan, China. Lancet (London, England) 2020;395:497506.

18. Del Valle DM, Kim-Schulze S, Huang $\mathrm{H}-\mathrm{H}$, et al. An inflammatory cytokine signature predicts COVID-19 severity and survival. Nature Medicine 2020;26:1636-1643.

19. Livanos AE, Jha D, Cossarini $F$, et al. Intestinal host response to SARS-CoV-2 infection and COVID-19 outcomes in patients with gastrointestinal symptoms. Gastroenterology 2021.

20. Everett J, Hokama P, Roche AM, et al. SARS-CoV-2 Genomic Variation in Space and Time in Hospitalized Patients in Philadelphia. mBio 2021;12:e03456-20.

21. Cheung KS, Hung IFN, Chan PPY, et al. Gastrointestinal Manifestations of SARS-CoV-2 Infection and Virus Load in Fecal Samples From a Hong Kong Cohort: Systematic Review and Meta-analysis. Gastroenterology 2020;159:8195.

22. Dong Z-Y, Xiang B-J, Jiang M, et al. The Prevalence of Gastrointestinal Symptoms, Abnormal Liver Function, Digestive System Disease and Liver Disease in COVID-19 Infection: A Systematic Review and Meta-Analysis. Journal of clinical gastroenterology 2021;55:67-76.

23. Choi B, Choudhary MC, Regan J, et al. Persistence and Evolution of SARS-CoV2 in an Immunocompromised Host. New England Journal of Medicine 2020;383:2291-2293.

24. Kemp SA, Collier DA, Datir RP, et al. SARS-CoV-2 evolution during treatment of chronic infection. Nature 2021.

25. Dorrell RD, Dougherty MK, Barash EL, et al. Gastrointestinal and hepatic manifestations of COVID-19: A systematic review and meta-analysis. JGH Open 2021;5:107-115.

26. Miller J, Cantor A, Zachariah P, et al. Gastrointestinal Symptoms as a Major Presentation Component of a Novel Multisystem Inflammatory Syndrome in Children That Is Related to Coronavirus Disease 2019: A Single Center Experience of 44 Cases. Gastroenterology 2020;159:1571-1574.e2.

27. Redd WD, Zhou JC, Hathorn KE, et al. Prevalence and Characteristics of Gastrointestinal Symptoms in Patients With Severe Acute Respiratory Syndrome Coronavirus 2 Infection in the United States: A Multicenter Cohort Study. Gastroenterology 2020;159:765-767.e2.

28. Sultan S, Altayar O, Siddique SM, et al. AGA Institute Rapid Review of the Gastrointestinal and Liver Manifestations of COVID-19, Meta-Analysis of International Data, and Recommendations for the Consultative Management of Patients with COVID-19. Gastroenterology 2020;159:320-334.e27. 
29. Lin L, Jiang X, Zhang Z, et al. Gastrointestinal symptoms of 95 cases with SARSCoV-2 infection. Gut 2020;69:997.

30. Trindade AJ, Izard S, Coppa K, et al. Gastrointestinal Bleeding in Hospitalized COVID-19 Patients: A Propensity Score Matched Cohort Study. J Intern Med 2020.

31. Norsa L, Bonaffini PA, Indriolo A, et al. Poor Outcome of Intestinal Ischemic Manifestations of COVID-19. Gastroenterology 2020;159:1595-1597.e1.

32. Jose M, Desai K. Fatal Superimposed Bacterial Sepsis in a Healthy Coronavirus (COVID-19) Patient. Cureus 2020;12:e8350-e8350.

33. Lansbury L, Lim B, Baskaran V, et al. Co-infections in people with COVID-19: a systematic review and meta-analysis. The Journal of infection 2020;81:266-275.

34. Valette X, du Cheyron D, Goursaud S. Mediastinal lymphadenopathy in patients with severe COVID-19. The Lancet Infectious Diseases 2020;20:1230.

35. Desai J, Patel U, Arjun S, et al. Impact of Liver Injury in COVID-19 Patients: Single-center Retrospective Cohort Analysis. J Clin Transl Hepatol 2020;8:476478.

36. Nardo AD, Schneeweiss-Gleixner M, Bakail M, et al. Pathophysiological mechanisms of liver injury in COVID-19. Liver international : official journal of the International Association for the Study of the Liver 2021;41:20-32.

37. Yang Y, Yang M, Yuan J, et al. Laboratory Diagnosis and Monitoring the Viral Shedding\&\#xa0; of SARS-CoV-2 Infection. The Innovation 2020;1.

38. Zou L, Ruan F, Huang M, et al. SARS-CoV-2 Viral Load in Upper Respiratory Specimens of Infected Patients. New England Journal of Medicine 2020;382:1177-1179.

39. Wölfel R, Corman VM, Guggemos W, et al. Virological assessment of hospitalized patients with COVID-2019. Nature 2020;581:465-469.

40. Young BE, Ong SWX, Kalimuddin S, et al. Epidemiologic Features and Clinical Course of Patients Infected With SARS-CoV-2 in Singapore. Jama 2020;323:1488-1494.

41. Landry ML, Criscuolo J, Peaper DR. Challenges in use of saliva for detection of SARS CoV-2 RNA in symptomatic outpatients. J Clin Virol 2020;130:104567.

42. Lu X, Wang L, Sakthivel SK, et al. US CDC Real-Time Reverse Transcription PCR Panel for Detection of Severe Acute Respiratory Syndrome Coronavirus 2. Emerg Infect Dis 2020;26:1654-65.

43. Huang N, Pérez P, Kato T, et al. SARS-CoV-2 infection of the oral cavity and saliva. Nature Medicine 2021.

44. United States Department of Agriculture APHIS. Confirmed Cases of SARS-CoV2 in Animals in the United States, 2020.

45. Daly N. Buddy, first dog to test positive for COVID-19 in the U.S., has died. National Geographic, 2020.

46. American Veterinary Medical Association 2017-2018 U.S. Pet Ownership \& Demographics Sourcebook. Volume https://www.avma.org/sites/default/files/resources/AVMA-Pet-DemographicsExecutive-Summary.pdf, 2018.

47. Zhang $\mathrm{Q}$, Zhang $\mathrm{H}$, Huang $\mathrm{K}$, et al. SARS-CoV-2 neutralizing serum antibodies in cats: a serological investigation. bioRxiv 2020:2020.04.01.021196. 
48. Patterson EI, Elia G, Grassi A, et al. Evidence of exposure to SARS-CoV-2 in cats and dogs from households in Italy. Nat Commun 2020;11:6231.

49. Fritz M, Rosolen B, Krafft E, et al. High prevalence of SARS-CoV-2 antibodies in pets from COVID-19+ households. One Health 2021;11:100192.

50. Braun KM, Moreno GK, Halfmann PJ, et al. Transmission of SARS-CoV-2 in domestic cats imposes a narrow bottleneck. PLOS Pathogens 2021;17:e1009373.

51. Michael HT, Waterhouse T, Estrada M, et al. Frequency of respiratory pathogens and SARS-CoV-2 in canine and feline samples submitted for respiratory testing in early 2020. J Small Anim Pract 2021.

52. Tyson JR, James P, Stoddart D, et al. Improvements to the ARTIC multiplex PCR method for SARS-CoV-2 genome sequencing using nanopore. bioRxiv 2020:2020.09.04.283077.

53. Shu Y, McCauley J. GISAID: Global initiative on sharing all influenza data - from vision to reality. Euro surveillance : bulletin Europeen sur les maladies transmissibles = European communicable disease bulletin 2017;22:30494. 